\title{
Likelihood of infection in patients with presumed sepsis at the time of intensive care unit admission: a cohort study
}

Peter M. C. Klein Klouwenberg ${ }^{1,2,3^{*}}$, Olaf L. Cremer ${ }^{1}$, Lonneke A. van Vught ${ }^{4}$, David S. Y. Ong ${ }^{1,2,3}$, Jos F. Frencken ${ }^{1,3}$, Marcus J. Schultz ${ }^{5}$ Marc J. Bonten ${ }^{2,3}$ and Tom van der Poll ${ }^{4}$

\begin{abstract}
Introduction: A clinical suspicion of infection is mandatory for diagnosing sepsis in patients with a systemic inflammatory response syndrome. Yet, the accuracy of categorizing critically ill patients presenting to the intensive care unit (ICU) as being infected or not is unknown. We therefore assessed the likelihood of infection in patients who were treated for sepsis upon admission to the ICU, and quantified the association between plausibility of infection and mortality.

Methods: We studied a cohort of critically ill patients admitted with clinically suspected sepsis to two tertiary ICUs in the Netherlands between January 2011 and December 2013. The likelihood of infection was categorized as none, possible, probable or definite by post-hoc assessment. We used multivariable competing risks survival analyses to determine the association of the plausibility of infection with mortality.

Results: Among 2579 patients treated for sepsis, 13\% had a post-hoc infection likelihood of "none", and an additional $30 \%$ of only "possible". These percentages were largely similar for different suspected sites of infection. In crude analyses, the likelihood of infection was associated with increased length of stay and complications. In multivariable analysis, patients with an unlikely infection had a higher mortality rate compared to patients with a definite infection (subdistribution hazard ratio 1.23; 95\% confidence interval 1.03-1.49).

Conclusions: This study is the first prospective analysis to show that the clinical diagnosis of sepsis upon ICU admission corresponds poorly with the presence of infection on post-hoc assessment. A higher likelihood of infection does not adversely influence outcome in this population.

Trial registration: ClinicalTrials.gov NCT01905033. Registered 11 July 2013.
\end{abstract}

\section{Introduction}

Sepsis is a syndrome that arises when the body's response to a severe infection injures its own tissues. In 1992 an international consensus panel proposed a clinical definition for sepsis, making use of the concept of a "systemic inflammatory response syndrome" (SIRS), involving alterations in body temperature, heart rate, respiration rate, and leukocyte counts [1]. The panel

\footnotetext{
* Correspondence: p.m.c.kleinklouwenberg@umcutrecht.nl

'Department of Intensive Care Medicine, University Medical Center Utrecht,

Room F06.149, P.O. Box 85500, 3508 GA, Utrecht, The Netherlands

${ }^{2}$ Department of Medical Microbiology, University Medical Center Utrecht, P.O. Box 85500, 3508 GA, Utrecht, The Netherlands

Full list of author information is available at the end of the article
}

defined sepsis as SIRS caused by suspected infection. It further introduced the terms severe sepsis to describe cases when sepsis is complicated by acute organ dysfunction and septic shock as severe sepsis complicated by hypotension refractory to fluid resuscitation. These definitions, generally referred to as the "Bone criteria", have been used as inclusion criteria in many clinical sepsis trials, and until today have remained largely unchanged [2].

Although the clinical suspicion of infection is a crucial factor in making a sepsis diagnosis, little is known about the accuracy of this diagnosis in the context of critically ill patients who present to the ICU with signs and symptoms of a "sepsis syndrome". We hypothesized that in 
the clinical practice of an ICU the diagnosis of sepsis is not based on strict diagnostic criteria for infection and that as a consequence the occurrence of sepsis on the ICU might be overestimated. Quantification of this discordance is helpful for estimating incidence rates in epidemiological studies and the possible reduction of antibiotic use.

To address this hypothesis we assessed the concordance between the prospective clinical sepsis diagnosis made by bedside physicians and the post-hoc diagnosis of infection made by clinical researchers using strict criteria. In addition, we assessed the association of the likelihood of infection with outcome.

\section{Methods}

\section{Study design and population}

This cohort study was incorporated in the Molecular Diagnosis and Risk Stratification of Sepsis (MARS) project in the mixed ICUs of two tertiary referral centers in the Netherlands [3, 4]. The local ethical committee approved the study with opt-out consent (Medisch Ethische Toetsingscommissie UMC Utrecht; number 10-056C; approval 16 June 2010). Participants were notified of the study in writing by a brochure provided at ICU admission with an attached opt-out card that could be completed by the patient or by his or her legal representative in case of unwillingness to participate. For the current study, we analyzed all first admissions of adult patients with a sepsis diagnosis who were admitted between January 2011 and December 2013, with an expected stay of $>24$ hours.

\section{Data collection and definitions}

Dedicated and trained observers prospectively collected relevant data from all patients. An infectious event was prospectively recorded when systemic antibiotics were started for therapeutic reasons by the attending physician, as described previously [3]. For this study, we included infections that were diagnosed before ICU admission or until 48 hours afterwards. Patients who were initially not suspected of having an infection at admission (i.e., those not receiving therapeutic antibiotics at ICU admission), but in whom therapeutic antibiotics were started later than 2 days after admittance because the positive cultures and the continuous presence of clinical symptoms from the time of admission onwards suggested an infection in retrospect, were also included in this study. The clinical research team in these cases dated the sepsis event to the start of clinical symptoms. We performed a sensitivity analysis in which we excluded infections starting more than 48 hours before admission, because we anticipated that the diagnostic likelihood of these infections differed from those starting directly prior to admission. Of note, patients receiving (only) prophylactic antibiotics were not assumed to have sepsis.

The plausibility of infection (none, possible, probable, definite) was determined post hoc, based on all available clinical, microbiological, and radiological evidence and according to the Centers for Disease Control and Prevention (CDC) and the International Sepsis Forum (ISF) criteria [5, 6]. This post-hoc assessment was used as the "gold standard" for infection in our study. In short, independent observers discussed all patients with (senior) critical care physicians and infection specialists in daily multidisciplinary meetings, and performed a confirmatory review of the medical record at the time of ICU discharge or death, including any postmortem findings. All diagnoses were therefore made after consensus and continuous data integrity checks. The precise definitions used to diagnose infection in the present study are described in a previous publication by our consortium [3].

SIRS criteria were defined as: temperature $<36.0^{\circ} \mathrm{C}$ or $>38.0{ }^{\circ} \mathrm{C}$ during at least 2 hours and 1 hour, respectively; white blood cell count $<4 \times 10^{9} / 1$ or $>12 \times 10^{9} /$ l, or $>10 \%$ immature (band) forms; heart rate $>90 /$ minute during at least 1 hour; respiratory rate $>20$ /minute during at least 1 hour, partial pressure of carbon dioxide $<32 \mathrm{mmHg}$, or mechanical ventilation $[1,7]$. Sepsis was defined as at least two SIRS criteria plus the clinical suspicion of infection by senior clinicians; we chose this "conventional" sepsis definition making use of the SIRS criteria to allow for interpretation in the context of the many previous sepsis trials using these criteria [2]. In addition, patients with "do not resuscitate" orders with clinical suspicion of sepsis, but without antibiotics, were classified as having sepsis. Criteria for organ failure included the following signs of organ hypoperfusion or dysfunction: areas of mottled skin; capillary refilling requiring 3 seconds or longer; urine output $<0.5 \mathrm{ml} / \mathrm{kg}$ for at least 6 hours, elevated creatinine, or renal replacement therapy [8]; lactate $>2 \mathrm{mmol} / \mathrm{l}$; abrupt change in mental status; abnormal electroencephalographic findings; platelet count $<100,000$ platelets/ml or disseminated intravascular coagulation; acute respiratory distress syndrome; and cardiac dysfunction, as defined by echocardiography or direct measurement of the cardiac index [9]. Shock was defined as the presence of severe sepsis plus the use of noradrenaline at a dose of $>0.1 \mu \mathrm{g} / \mathrm{kg} /$ minute during at least $50 \%$ of the day. ICU-related complications such as ICU-acquired infections, acute kidney injury, and adult respiratory distress syndrome that were present at or occurred during ICU admission were prospectively registered $[10,11]$. For ICU-acquired infections, only infections that started $>48$ hours after admission with a probability of at least "possible" were included. 
All patients with sepsis were managed according to locally adapted protocols based on the Surviving Sepsis Campaign guidelines [12].

\section{Statistical analyses}

All results are presented as median and interquartile range (IQR) or number and percentage, as appropriate. Continuous nonparametric data were analyzed using a Kruskal-Wallis test and categorical data were analyzed using the chi-squared test. The Cochran-Armitage test for trend and the Gray's test for equality of cumulative incidence functions were used.

We assessed the effect of infection plausibility on ICU mortality using a competing risks survival analysis to account for informative censoring [13]. A competing risks analysis provides two measures of association: the causespecific hazard ratio (CSHR), which estimates the direct effects of infection on outcome (both ICU discharge and death); and the subdistribution hazard ratio (SHR), which is a summary measure of all separate causespecific hazards and can be used to calculate the cumulative incidence of the outcome of interest (i.e., death in this study) [14]. The plausibility of infection was included as a dichotomous variable (none/possible vs. probable/definite). We adjusted for confounders that were chosen a priori based on their expected associations with infection and mortality after careful consideration of the literature and based on clinical expertise. These included age, gender, cardiovascular disease, immunocompromised state, malignancy, diabetes mellitus, respiratory insufficiency, renal insufficiency, recent surgery, sepsis severity, site of infection, and Acute Physiology and Chronic Health Evaluation (APACHE) IV score. $p<0.05$ was considered statistically significant. All analyses were performed using SAS 9.2 (SAS Institute, Cary, NC, USA) and $\mathrm{R}$ version 3.10 [15].

\section{Results}

\section{Demographics}

Over the 3-year observation period we studied 6944 patients during 7347 hospitalizations contributing a total of 8259 ICU episodes, of which 912 ICU readmissions were excluded from analysis. At admission, 2738 patients $(37 \%)$ received therapeutic antimicrobials for a clinical suspicion of infection, of whom 2579 (94 \%) had at least two SIRS criteria and were thus diagnosed with sepsis. Table 1 presents the main characteristics of patients presenting to the ICU with a clinical diagnosis of sepsis. C-reactive protein and core temperature increased significantly with increasing likelihoods of infection, in contrast to leukocyte counts. The most frequent suspected primary sources of sepsis in the retrospective review of cases were pulmonary (community-acquired and hospital-acquired pneumonia, $n=1292$ ), abdominal (peritonitis, $n=414$ ), bloodstream (endocarditis, primary bloodstream, and catheter-related bloodstream, $n=230$ ), urinary tract $(n=162)$, and skin or soft tissue $(n=118)$ infections. The remaining 363 patients had infections at other sites.

\section{Accuracy of infection diagnosis}

Of all patients treated for sepsis, $13 \%$ had an infection likelihood of "none" upon post-hoc analysis (Table 1). An additional $30 \%$ had an infection likelihood of possible, whereas slightly more than half scored a higher infection likelihood ( $25 \%$ probable and $33 \%$ definite). Limiting the analysis to infections that were diagnosed within 48 hours before admission resulted in a similar distribution ( $n=2117): 15 \%, 32 \%, 25 \%$, and $28 \%$ were classed as none, possible, probable, and definite infections, respectively. Figure 1 shows the plausibility of infection after post-hoc analysis for the whole cohort, and stratified by sepsis severity. Although the accuracy of the infection diagnosis according to the post-hoc adjudication increased with greater sepsis severity, there was still considerable misclassification in patients with organ failure (40\% of patients classified as none or possible) or shock (34\%). Figure 2 shows the plausibility of infection after post-hoc analysis for the five most prevalent sources of infection. The proportion of definite and probable infections was largely similar in patients with different sources of infection, although the percentage of definite cases in pneumonia patients was significantly lower compared with the whole cohort (16 \% vs. $33 \%$, $p<0.001)$. Furthermore, there were no likelihoods of "none" in the cases with skin or soft tissue infection. Additional file 1 shows all (both sepsis and nonsepsis) diagnoses that were registered in patients by category of infection likelihood.

\section{Outcomes}

Figure 3 shows various patient outcomes in the whole population, and stratified by infection likelihood and the most prevalent presumed sources of infection. The plausibility of infection was not associated with mortality either in the entire patient population admitted with a sepsis diagnosis $(21 \%, 18 \%, 20 \%$, and $20 \%$ mortality in patients with infection likelihoods of none, possible, probable, and definite, respectively) or in any of the main subgroups of presumed infection sites except for the lungs. Figure 4 displays the cumulative incidence functions of mortality for the none-possible vs. probable-definite classes of infection plausibility. The confidence intervals for all four categories overlap, meaning that in this crude survival analysis plausibility of infection was also not associated with mortality ( $p=0.73$; crude SHR 1.05; 95 \% confidence interval (CI) 0.88-1.25). In the multivariable analysis, however, a higher plausibility of 
Table 1 Baseline characteristics of patients admitted with presumed sepsis

\begin{tabular}{|c|c|c|c|c|c|c|}
\hline & \multirow[t]{2}{*}{ All } & \multicolumn{5}{|c|}{ Post-hoc plausibility of infection } \\
\hline & & None & Possible & Probable & Definite & $p$ value \\
\hline Number & $2579(100 \%)$ & $332(13 \%)$ & $771(30 \%)$ & $633(25 \%)$ & $843(33 \%)$ & $n / a$ \\
\hline \multicolumn{7}{|l|}{ Demographics } \\
\hline Age (years) & $62(49,71)$ & $62(48,72)$ & $62(51,71)$ & $62(49,71)$ & $62(49,71)$ & 0.73 \\
\hline Gender, male & $1540(60 \%)$ & $181(55 \%)$ & $493(64 \%)$ & $365(58 \%)$ & 501 (59 \%) & 0.04 \\
\hline Race, Caucasian & 2257 (88 \%) & $278(84 \%)$ & $688(89 \%)$ & $552(87 \%)$ & $739(88 \%)$ & 0.09 \\
\hline Body mass index $>30 \mathrm{~kg} / \mathrm{m}^{2}$ & $479(19 \%)$ & $53(16 \%)$ & $166(22 \%)$ & $93(15 \%)$ & $167(20 \%)$ & 0.004 \\
\hline \multicolumn{7}{|l|}{ Comorbidities } \\
\hline Charlson comorbidity index & $3.5(0,9.1)$ & $1.5(0,8.1)$ & $2.5(0.0,9.4)$ & $4.6(0.0,9.4)$ & $4.6(0.0,9.7)$ & 0.02 \\
\hline Cardiovascular disease $^{a}$ & $605(23 \%)$ & $81(24 \%)$ & $204(26 \%)$ & $142(22 \%)$ & $178(21 \%)$ & 0.07 \\
\hline Respiratory insufficiency ${ }^{b}$ & $432(17 \%)$ & $50(15 \%)$ & $141(18 \%)$ & $120(19 \%)$ & $121(14 \%)$ & 0.05 \\
\hline Renal insufficiency ${ }^{c}$ & $329(13 \%)$ & 31 (9 \%) & $98(13 \%)$ & $70(11 \%)$ & $130(15 \%)$ & 0.02 \\
\hline Malignancy $^{d}$ & 239 (9 \%) & $20(6 \%)$ & $61(8 \%)$ & $68(11 \%)$ & $90(11 \%)$ & 0.02 \\
\hline Immunocompromised state ${ }^{e}$ & $628(24 \%)$ & $68(20 \%)$ & $159(21 \%)$ & $173(27 \%)$ & $228(27 \%)$ & 0.002 \\
\hline Diabetes mellitus & $486(19 \%)$ & $63(19 \%)$ & $143(19 \%)$ & $119(19 \%)$ & $161(19 \%)$ & 0.99 \\
\hline \multicolumn{7}{|l|}{ Admission characteristics } \\
\hline Surgical admission & $661(26 \%)$ & $88(27 \%)$ & 186 (24 \%) & 122 (19 \%) & 265 (31\%) & $<0.001$ \\
\hline APACHE IV score & $77(66,100)$ & $74(58,101)$ & $75(58,96)$ & $79(61,100)$ & $79(60,101)$ & 0.08 \\
\hline Core temperature & $37.8(37.0,38.6)$ & $37.6(36.9,38.4)$ & $37.7(37.0,38.5)$ & $37.9(37.1,38.6)$ & $37.9(37.1,38.7)$ & $<0.001$ \\
\hline White blood cell count & $14.2(9.6,19.8)$ & $13.5(9.9,18.8)$ & $14.6(10.4,19.0)$ & $14.2(9.6,20.1)$ & $14.5(8.5,20.5)$ & 0.88 \\
\hline C-reactive protein & $114(35,229)$ & $36(8,102)$ & $86(19,181)$ & $125(47,234)$ & $170(78,270)$ & $<0.001$ \\
\hline Creatinine & $104(70,171)$ & $101(68,167)$ & $100(69,157)$ & $94(65,158)$ & $118(75,198)$ & $<0.001$ \\
\hline Sepsis severity at admission & & & & & & $<0.001$ \\
\hline Sepsis & 1076 (42\%) & $175(53 \%)$ & 380 (49 \%) & $238(38 \%)$ & $283(34 \%)$ & \\
\hline Severe sepsis & $727(28 \%)$ & 70 (21 \%) & $224(29 \%)$ & 198 (31\%) & $235(28 \%)$ & \\
\hline Septic shock & $776(30 \%)$ & 87 (26 \%) & $167(22 \%)$ & $197(31 \%)$ & $325(39 \%)$ & \\
\hline \multicolumn{7}{|l|}{ Organ failure at admission ${ }^{f}$} \\
\hline Central nervous system & $0.0(0.0,1.0)$ & $0.0(0.0,1.0)$ & $0.0(0.0,1.0)$ & $0.0(0.0,1.0)$ & $0.0(0.0,1.0)$ & 0.22 \\
\hline Cardiovascular & $3.0(1.0,4.0)$ & $3.0(1.0,4.0)$ & $3.0(1.0,4.0)$ & $3.0(1.0,4.0)$ & $3.0(1.0,4.0)$ & $<0.001$ \\
\hline Respiratory & $2.5(2.0,3.0)$ & $3.0(2.0,3.0)$ & $3.0(2.0,3.0)$ & $3.0(2.0,3.0)$ & $2.0(2.0,3.0)$ & 0.35 \\
\hline Renal & $0.0(0.0,2.0)$ & $0.0(0.0,1.0)$ & $0.0(0.0,1.0)$ & $0.0(0.0,1.0)$ & $1.0(0.0,3.0)$ & $<0.001$ \\
\hline Hepatic & $0.0(0.0,0.0)$ & $0.0(0.0,0.0)$ & $0.0(0.0,0.0)$ & $0.0(0.0,0.0)$ & $0.0(0.0,0.0)$ & $<0.001$ \\
\hline Coagulation & $0.0(0.0,1.0)$ & $0.0(0.0,1.0)$ & $0.0(0.0,1.0)$ & $0.0(0.0,1.0)$ & $0.0(0.0,1.5)$ & $<0.001$ \\
\hline Total & $7.0(5.0,10)$ & $7.0(5.0,10)$ & $7.0(4.0,9.0)$ & $7.0(5.0,9.0)$ & $8.0(5.0,11)$ & $<0.001$ \\
\hline \multicolumn{7}{|l|}{ Treatment at admission } \\
\hline Mechanical ventilation & 2016 (78 \%) & $261(79 \%)$ & 608 (79 \%) & 492 (78 \%) & 655 (78 \%) & 0.93 \\
\hline Dialysis & $263(10 \%)$ & 39 (12 \%) & $67(9 \%)$ & 54 (9 \%) & 103 (12\%) & 0.037 \\
\hline
\end{tabular}

Data presented as median (interquartile range) or number (\%). The four infection plausibility classes were compared using the Kruskal-Wallis test or the chi-squared test

${ }^{a}$ Cardiovascular disease was defined as cerebrovascular disease or chronic cardiovascular insufficiency (New York Heart Association class 4), chronic congestive heart failure (ejection fraction $<30 \%$ ), or peripheral vascular disease (intermittent claudication, patients with percutaneous transluminal angioplasty, or bypass for arterial insufficiency)

${ }^{b}$ Respiratory insufficiency was defined as chronic obstructive pulmonary disease or chronic respiratory insufficiency with functional disabilities (chronic mechanical ventilation, oxygen use at home, or severe pulmonary hypertension)

${ }^{c}$ Renal insufficiency was defined as chronic renal insufficiency (creatinine $>177 \mu \mathrm{mol} / \mathrm{l}$ ) or chronic dialysis

${ }^{\mathrm{d}}$ Malignancy included both metastatic and hematologic malignancies

eImmunocompromised state was defined as having acquired immunodeficiency syndrome, the use of corticosteroids in high doses (equivalent to prednisolone of $>75 \mathrm{mg}$ /day for at least 1 week), current use of immunosuppressive drugs, current use of antineoplastic, drugs recent hematologic malignancy, or documented humoral or cellular deficiency

Based on the Sequential Organ Failure Assessment scores

APACHE Acute Physiology and Chronic Health Evaluation, n/a not applicable 


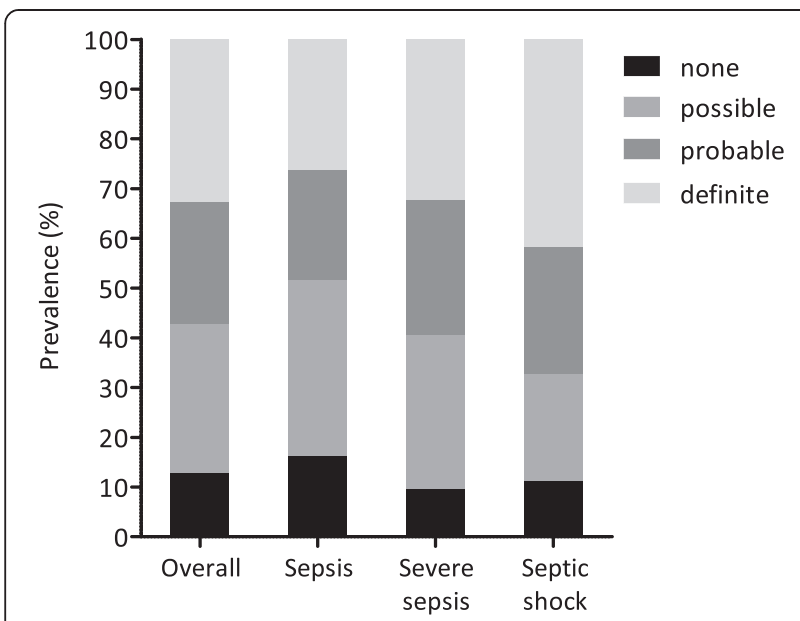

Fig. 1 Plausibility of infection stratified by clinical severity upon presentation in patients with presumed sepsis. Comparison between the clinical diagnosis of infection at the time of ICU admission and the actual presence of infection as determined by post-hoc evaluation

infection (probable/definite) was associated with a lower mortality (SHR 0.81; $95 \%$ CI 0.67-0.97). This means that patients with a confirmed infection diagnosis actually have a lower mortality rate than patients with an unconfirmed infection or an alternative diagnosis. Cause-specific analysis revealed that this reduction was caused by a direct effect on death (CSHR 0.73; 95 \% CI 0.61-0.89), and not by the indirect effect on a longer ICU length of stay (CSHR 0.93; $95 \%$ CI 0.85-1.02). In subgroup analyses, the mortality hazard for each hospital was similar (hospital A: SHR 0.80, 95 \% CI 0.62-1.03; hospital B: SHR 0.85, 95 \% CI 0.63-1.13). These estimates were similar when restricting our analysis to cases with none or definite infections only (SHR 0.75, $95 \%$ CI 0.55-1.01). Furthermore, the

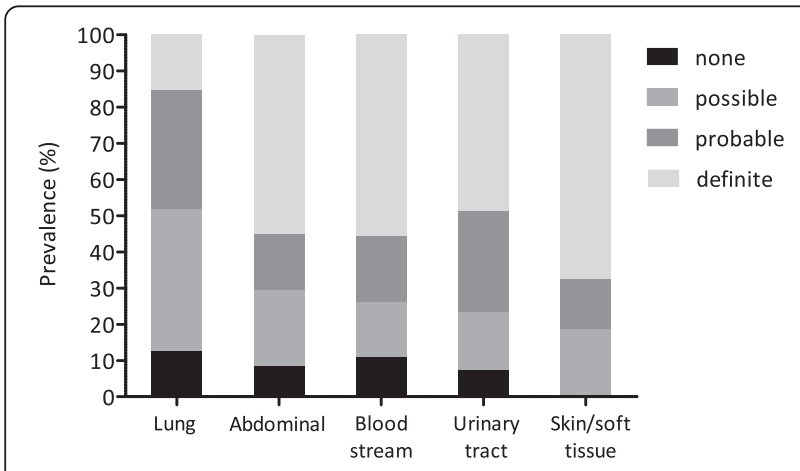

Fig. 2 Plausibility of infection in patients with presumed sepsis upon presentation for the most frequent sites of infection. Distribution of plausibility of infection for lung infections (community-acquired pneumonia and hospital-acquired pneumonia), abdominal infections (primary and secondary peritonitis), bloodstream infections (primary bloodstream infections, catheter-related bloodstream infections, and endocarditis), urinary tract infections, and skin/soft tissue infections prevalence of the adult respiratory distress syndrome, the prevalence of acute kidney injury, and the length of stay significantly increased with greater infection likelihoods $(p<0.001)$, whereas the occurrence of ICUacquired infections did not $(p=0.36)$ (Fig. 3). In the main subgroups of presumed infection sites, the infection plausibility was not associated with outcome parameters in this crude analysis, except for pulmonary infections.

\section{Discussion}

We determined the accuracy of the infection diagnosis made by clinicians in the context of presumed sepsis upon admission to the ICU and found that up to $43 \%$ of patients treated for sepsis were unlikely to have had an infection on post-hoc assessment. Although the accuracy of the infection diagnosis increased with increasing severity of disease, a considerable proportion of patients with severe sepsis and septic shock still had at most a possible infection. These results show that making an accurate infection diagnosis upon ICU admission in patients with suspected sepsis is difficult in many cases.

Our study is the first prospective comparison of sepsis diagnoses made by ICU physicians and post-hoc analyses of infection likelihoods based on strict diagnostic criteria, revealing that the true incidence of sepsis upon ICU admission is probably overestimated. Only few previous studies have specifically investigated the accuracy of infection diagnoses in patients with suspected sepsis in the ICU. A French study found that $49 \%$ of patients were potentially unnecessarily treated for a new infection on the ICU [16]. This finding was based on the level of microbiological evidence and not on well-defined diagnostic criteria, however, making it difficult to appreciate the true percentage of patients without infection in post-hoc analysis. Another study explored the correlation of clinical certainty at the start of antimicrobial therapy with the post-hoc presence of infection [17]. The primary aim of this latter investigation focused on antimicrobial use, namely how often administration of antimicrobials for suspected infection could be justified by the presence of infection; a large proportion of patients treated with empirical antibiotics (58 of the 125; $46 \%$ ) actually had no infection according to the infectious diseases specialist in the post-hoc assessment [17].

In crude analysis, the likelihood of infection in patients treated for suspected sepsis was not associated with mortality. Since several factors that impact on ICU mortality were unequally distributed between groups, we performed multivariable survival analysis and found that a lower likelihood of infection was associated with increased mortality. In other words, patients who were initially treated for sepsis but had, in retrospect, a 


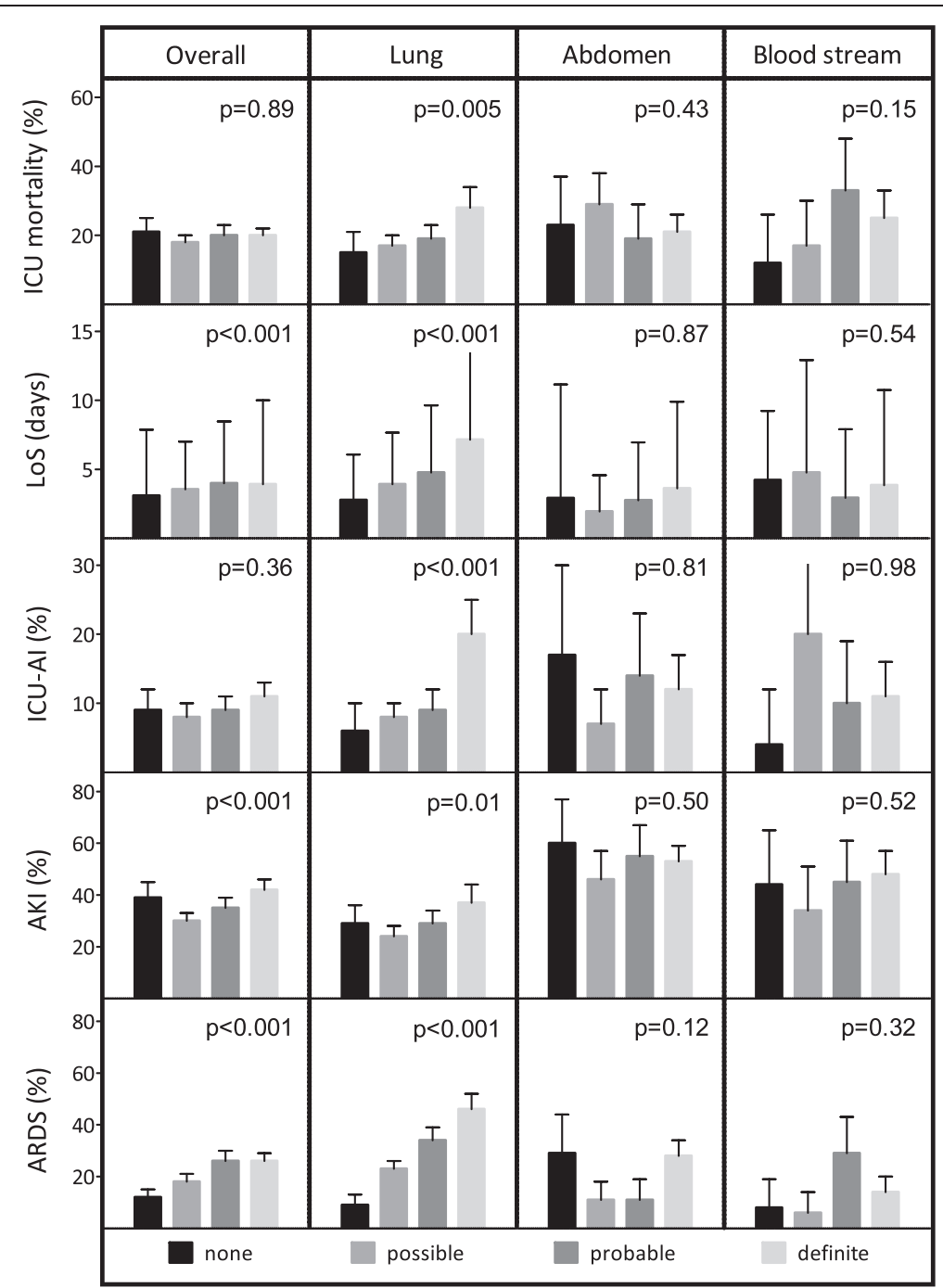

Fig. 3 Patient outcomes for various sites of infection stratified by plausibility of infection. Data are crude associations. The length of ICU stay (LoS) is shown as median. ICU-acquired infections (ICU-A) were defined as infections that started $>48$ hours after admission with a plausibility of infection of at least possible. Acute kidney injury (AKI) and adult respiratory distress syndrome (ARDS) that were present at or occurred during ICU admission were taken into account. Whiskers indicate the $95 \% \mathrm{Cl}$. p values indicate the results of the Cochran-Armitage chi-square test for trend. Urinary tract and skin/soft tissue infections are not shown because of relatively small subgroups after stratification

noninfectious diagnosis had a higher mortality rate compared with patients with an infection. This observation is probably related to variations in underlying pathology, but may also partly be due to the diagnostic delay that resulted from an incorrect working diagnosis. Furthermore, these data suggest that infection does not result in worse outcome compared with other critical conditions. It is important to note that the increasing incidences of complications such as acute kidney injury and adult respiratory distress syndrome in cases with higher plausibilities of infection are markers of a "correct" sepsis diagnosis and should not be interpreted as causal factors of the lower adjusted mortality rates in noninfectious cases.
As the discrimination between infectious and noninfectious causes of critical illness in the ICU using clinical parameters only has proved challenging, multiple studies have been performed into the value of other markers, such as host biomarkers for the diagnosis of infection [18, 19]. While some biomarkers, such as procalcitonin, may aid in limiting the duration of antibiotic therapy in ICU patients [20], at present there are no biomarkers that provide sufficient diagnostic accuracy to withhold antibiotics as initial therapy in ICU patients with suspected infection $[18,21,22]$. While biomarkers would be valuable for diagnosis in reducing antibiotic use in this patient population, our current study suggests that 


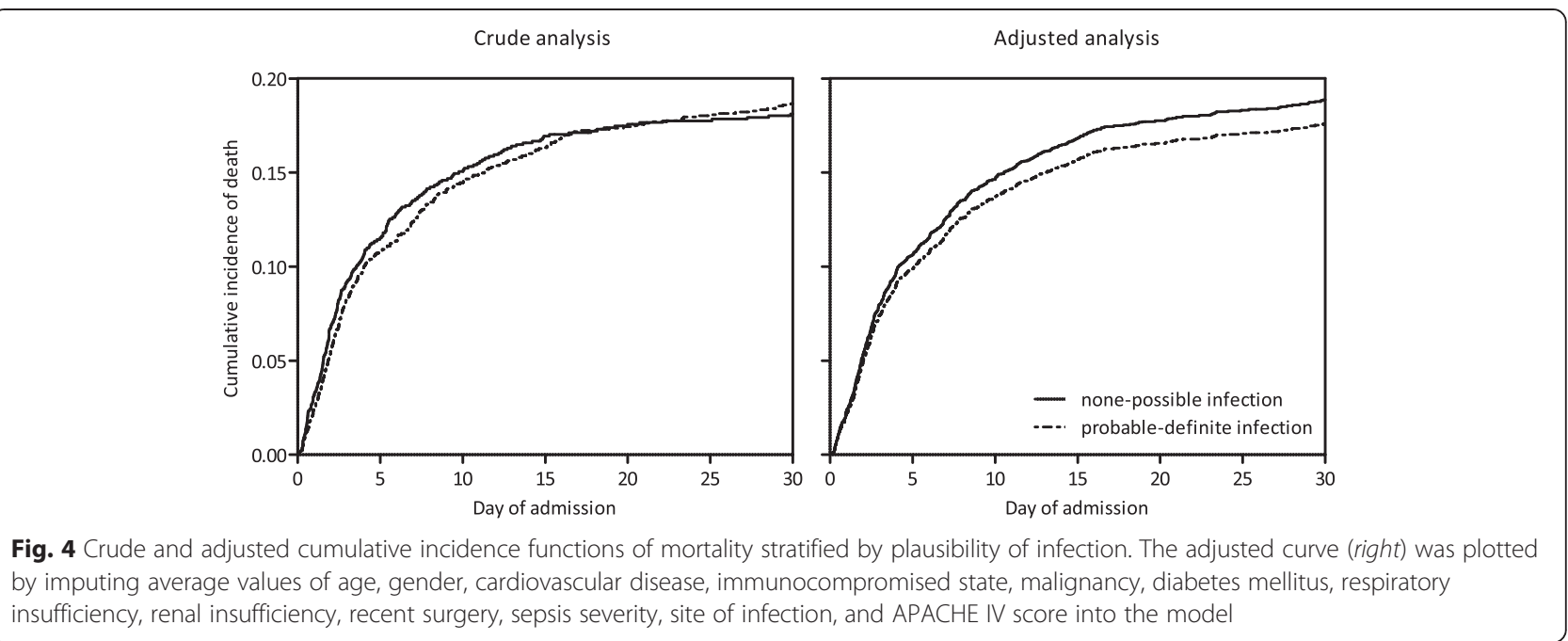

for stratification according to risk for an adverse outcome, the infection diagnosis itself is less important.

A limitation of this study concerns the inherently somewhat complex CDC and ISF infection definitions used for the post-hoc assessment for the presence of infection. We therefore determined the diagnostic agreement among the study team in a separate study, and concordance was found to be good [3]. In contrast to this previous study, the current process of prospective surveillance involved discussions among observers, discussions with (senior) clinicians in multidisciplinary meetings attended by critical care physicians and infection specialists, and continuous checks of data integrity. All diagnoses were therefore made after consensus. As such, our post-hoc analyses represent an "ideal" situation with availability of all diagnostic data collected after the acute event. Consequently, our study should not be interpreted as an analysis of the adequacy of clinical action in the ICU, but rather as an attempt to assess the true incidence of infection in patients admitted with suspected sepsis. In this respect it is important to note that in large surveys the rapid administration of broadspectrum antibiotics to patients with clinically diagnosed septic shock is associated with a time-dependent increase in survival $[23,24]$, suggesting that the benefit of early antibiotic treatment in patients with infection is greater than the potential harm of unnecessary antimicrobial therapy in those without infection. Notably, relative to other sources of infection, only few pneumonia cases fulfilled the criteria for definite infection. This was most probably caused by a relatively strict definition for pneumonia [3]. Furthermore, any systemic use of antibiotics before admission may have influenced culture results obtained in the ICU and therefore also the recorded likelihood of infection. However, a positive culture was not necessarily needed to diagnose a probable infection, including for the most commonly observed community-acquired infection in our study (pneumonia). For patients with hospital-acquired infections, this issue was deemed less problematic since blood (and other) cultures were typically collected before the start of antibiotics. Another limitation involves the fact that this study was performed in two centers in the Netherlands and may not reflect general ICU practice. Lastly, as is true for all observational studies, we cannot rule out the possibility that unobserved confounding might have occurred in the mortality analysis. However, the adjustment methods used were identical for all subgroups.

\section{Conclusions}

This first prospective analysis of the accuracy of the infection diagnosis in patients with suspected sepsis on ICU admission shows that the clinical diagnosis of sepsis corresponds poorly with the actual presence of infection, as defined by CDC/ISF diagnostic criteria. These results suggest that the true incidence of sepsis may have been overestimated in many studies. In fact, a substantial portion of patients being enrolled in clinical sepsis trials may in fact not have probable or definite infection, which may negatively impact the power of such trials to show benefit of certain sepsis treatments.

\section{Key messages}

- The clinical diagnosis of sepsis on admission corresponds poorly with the presence of infection defined by strict diagnostic criteria.

- A higher likelihood of infection does not negatively impact the mortality of patients treated for sepsis. 


\section{Additional file}

Additional file 1: Is Table S1 presenting diagnoses in patients admitted with sepsis by infection likelihood. Overview of all (both sepsis and nonsepsis) diagnoses that were recorded in patients upon admission by category of infection likelihood. (PDF $144 \mathrm{~kb}$ )

\section{Abbreviations}

APACHE: Acute Physiology and Chronic Health Evaluation; CDC: Centers for Disease Control and Prevention; Cl: Confidence interval; CSHR: Cause-specific hazard ratio; IQR: Interquartile range; ISF: International Sepsis Forum; MARS: Molecular Diagnosis and Risk Stratification of Sepsis; SHR: Subdistribution hazard ratio; SIRS: Systemic inflammatory response syndrome.

\section{Competing interests}

All authors declare that no conflicts of interest exist. The sponsors did not play a role in the design and conduct of the study; collection, management, analysis, and interpretation of the data; and preparation, review, or approval of the manuscript; and decision to submit the manuscript for publication.

\section{Authors' contributions}

PMCKK, OLC, and TVdP substantially contributed to the conception and design of this study. PMCKK, OLC, LAvV, DSYO, JFF, MJS, MJB, and TVdP acquired the data. PMCKK had full access to all data in the study and takes responsibility for the integrity of the data and the accuracy of the data analysis. PMCKK, OLC, and TVdP were involved in the interpretation of data. PMCKK, OLC and TPvd drafted the manuscript and all authors revised it critically for important intellectual content. All authors gave final approval of this version to be submitted.

\section{Acknowledgements}

This work was supported by the Center for Translational Molecular Medicine (http://www.ctmm.nl) project MARS (grant 04I-201). MJB received research funding from the Netherlands Organization of Scientific Research (NWO Vici 918.76.611).

\section{Author details}

${ }^{1}$ Department of Intensive Care Medicine, University Medical Center Utrecht, Room F06.149, P.O. Box 85500, 3508 GA, Utrecht, The Netherlands. ${ }^{2}$ Department of Medical Microbiology, University Medical Center Utrecht, P.O. Box 85500, 3508 GA, Utrecht, The Netherlands. ${ }^{3}$ Julius Center for Health Sciences and Primary Care, University Medical Center Utrecht, P.O. Box 85500, 3508 GA, Utrecht, The Netherlands. ${ }^{4}$ Center for Experimental and Molecular Medicine and Division of Infectious Diseases, Academic Medical Center, University of Amsterdam, Meibergdreef 9, 1105 AZ, Amsterdam, The Netherlands. ${ }^{5}$ Department of Intensive Care Medicine, Academic Medical Center, University of Amsterdam, Meibergdreef 9, 1105 AZ, Amsterdam, The Netherlands.

Received: 30 May 2015 Accepted: 15 August 2015

Published online: 07 September 2015

\section{References}

1. Bone RC, Balk RA, Cerra FB, Dellinger RP, Fein AM, Knaus WA, et al. Definitions for sepsis and organ failure and guidelines for the use of innovative therapies in sepsis. The ACCP/SCCM Consensus Conference Committee. American College of Chest Physicians/Society of Critical Care Medicine. Chest. 1992;101:1644-55.

2. Angus DC, van der Poll T. Severe sepsis and septic shock. N Engl J Med. 2013;369:840-51.

3. Klein Klouwenberg PM, Ong DS, Bos LD, de Beer FM, van Hooijdonk RT, Huson MA, et al. Interobserver agreement of Centers for Disease Control and Prevention criteria for classifying infections in critically ill patients. Crit Care Med. 2013;41:2373-8.

4. Klein Klouwenberg PM, van Mourik MS, Ong DS, Horn J, Schultz MJ, Cremer $\mathrm{OL}$, et al. Electronic implementation of a novel surveillance paradigm for ventilator-associated events. Feasibility and validation. Am J Respir Crit Care Med. 2014;189:947-55.
5. Horan TC, Andrus M, Dudeck MA. CDC/NHSN surveillance definition of health care-associated infection and criteria for specific types of infections in the acute care setting. Am J Infect Control. 2008;36:309-32.

6. Calandra T, Cohen J. The international sepsis forum consensus conference on definitions of infection in the intensive care unit. Crit Care Med. 2005;33:1538-48

7. Levy MM, Fink MP, Marshall JC, Abraham E, Angus D, Cook D, et al. 2001 SCCM/ESICM/ACCP/ATS/SIS International Sepsis Definitions Conference. Crit Care Med. 2003;31:1250-6.

8. Bellomo R, Ronco C, Kellum JA, Mehta RL, Palevsky P. Acute renal failure-definition, outcome measures, animal models, fluid therapy and information technology needs: the Second International Consensus Conference of the Acute Dialysis Quality Initiative (ADQI) Group. Crit Care. 2004;8:R204-12.

9. Annane D, Bellissant E, Cavaillon JM. Septic shock. Lancet. 2005;365:63-78.

10. Mehta RL, Kellum JA, Shah SV, Molitoris BA, Ronco C, Warnock DG, et al. Acute Kidney Injury Network: report of an initiative to improve outcomes in acute kidney injury. Crit Care. 2007;11:R31.

11. Bernard GR, Artigas A, Brigham KL, Carlet J, Falke K, Hudson L, et al. The American-European Consensus Conference on ARDS. Definitions, mechanisms, relevant outcomes, and clinical trial coordination. Am J Respir Crit Care Med. 1994;149(3 Pt 1):818-24.

12. Dellinger RP, Levy MM, Rhodes A, Annane D, Gerlach H, Opal SM, et al. Surviving Sepsis Campaign: international guidelines for management of severe sepsis and septic shock, 2012. Intensive Care Med. 2013;39:165-228.

13. Wolkewitz M, Vonberg R, Grundmann H, Beyersmann J, Gastmeier P, Bärwolff $S$, et al. Risk factors for the development of nosocomial pneumonia and mortality on intensive care units: application of competing risks models. Crit Care. 2008;12:R44.

14. Fine JP, Gray RJ. A proportional hazards model for the subdistribution of a competing risk. J Am Stat Assoc. 1999;94:496-509.

15. A Language and Environment for Statistical Computing, R Core Team, R Foundation for Statistical Computing, Vienna, Austria, 2015, www.R-project.org. Accessed 27-08-2015.

16. Montravers P, Dupont H, Gauzit R, Veber B, Bedos JP, Lepape A. Strategies of initiation and streamlining of antibiotic therapy in 41 French intensive care units. Crit Care. 2011;15:R17.

17. Levin PD, Idrees S, Sprung CL, Weissman C, Weiss $Y$, Moses AE, et al. Antimicrobial use in the ICU: indications and accuracy-an observational trial. J Hosp Med. 2012;7:672-8.

18. Pierrakos C, Vincent JL. Sepsis biomarkers: a review. Crit Care. 2010;14:R15.

19. Bloos F, Reinhart K. Rapid diagnosis of sepsis. Virulence. 2014:5:154-60.

20. Bouadma L, Luyt CE, Tubach F, Cracco C, Alvarez A, Schwebel C, et al. Use of procalcitonin to reduce patients' exposure to antibiotics in intensive care units (PRORATA trial): a multicentre randomised controlled trial. Lancet. 2010;375:463-74.

21. Wacker C, Prkno A, Brunkhorst FM, Schlattmann P. Procalcitonin as a diagnostic marker for sepsis: a systematic review and meta-analysis. Lancet Infect Dis. 2013;13:426-35.

22. Wu Y, Wang F, Fan X, Bao R, Bo L, Li J, et al. Accuracy of plasma sTREM-1 for sepsis diagnosis in systemic inflammatory patients: a systematic review and meta-analysis. Crit Care. 2012;16:R229.

23. Ferrer R, Martin-Loeches I, Phillips G, Osborn TM, Townsend S, Dellinger RP, et al. Empiric antibiotic treatment reduces mortality in severe sepsis and septic shock from the first hour: results from a guideline-based performance improvement program. Crit Care Med. 2014;42:1749-55.

24. Kumar A, Roberts D, Wood KE, Light B, Parrillo JE, Sharma S, et al. Duration of hypotension before initiation of effective antimicrobial therapy is the critical determinant of survival in human septic shock. Crit Care Med. 2006;34:1589-96. 